International Mathematical Forum, 2, 2007, no. 48, 2379 - 2390

\title{
On the Existence of Solutions of Nonlinear Boundary Value Dynamic Systems with Linear Part on Time Scales
}

\author{
I. B. Yașar \\ Department of Mathematics \\ Faculty of Sciences and Arts \\ University of Gazi \\ Beșevler, 06500, Ankara, Turkey \\ irfany@gazi.edu.tr \\ M. T. Dastjerdi \\ Department of Mathematics \\ Faculty of Sciences, Unevirsity of Zanjan \\ Zanjan 45195-313, Iran \\ tdast@yahoo.com
}

\begin{abstract}
In this article we consider the existence of solution for the time scale system $y^{\Delta}=A(t) y(t)+F(t, y(t))$ for $t \in\left[t_{0}, t_{1}\right]_{T}$ with

$$
M y\left(t_{0}\right)+N y\left(t_{1}\right)=\alpha
$$

which can be extended to delta case and problem $y^{\Delta}=A(t) y(t)+$ $F\left(t, \rho(y(t))\right.$ with the same conditions. Here $A:\left[t_{0}, t_{1}\right] \rightarrow B\left(R^{n}\right)$ is ld-continuous and regressive and $M$ and $N$ square matrix of order $n$. We consider necessary and sufficient conditions for the existence of a solution to $y^{\Delta}=A(t) y(t)+f(t)$ with $(*)$ is that if $D=M \Phi_{A}\left(t_{0}, t_{0}\right)+$ $N \Phi_{A}\left(t_{1}, t_{0}\right)$ is nonsingular.
\end{abstract}

Mathematics Subject Classification: 37N35, 93D20

Keywords: Time scales; Time varying; Non Linear system; Two point boundary values problem, Multipoint boundary values problems 


\section{Introduction}

In recent years, a theory known as dynamic systems on time scales has been built which incorporates both continuous and discrete times, namely, time as an arbitrary closed set of reals, and permit us to handle both systems simultaneously [1]. This theory allows one to get some insight into and better understanding of subtle differences between discrete and continuous systems. Two point boundary values problems (TPBVP) play an important role in the theory of differential and difference equations and in the various applications of this theory, in particular, in problems of optimal control $[2,3]$. Up to this time, TPBVP for systems in sense of various forms of applications and several computational methods have been developed for solving TPBVP's $[4,5,6]$.

In this article, we have mentioned the needed definitions and theorems, then as a main of result, we obtain and prove solution of time varying dynamic systems with TPBVP on arbitrary time scales which can be extended to multi point BVP separated and non separated cases.

\section{Preliminaries}

First we give some aspects about Time Scales, then some needed lemmas and theorems are mentioned. The material in this section is drawn mainly from [1] and $[6]$.

\subsection{Time Scales}

A time scale is any nonempty closed subset of the real numbers $\mathbf{R}$. Thus time scale can be any of the usual integer subsets (e.g. $\mathbf{Z}$ or $\mathbf{N}$ ), the entire real line $\mathrm{R}$, or any combination of discrete points of union with continuous intervals.

The forward jump operator of $T, \sigma(t): T \rightarrow T$, is given by $\sigma(t)=$ $\inf _{s \in T}\{s>t\}$. The backward jump operator of $T, \rho(t): T \rightarrow T$, is given by $\rho(t)=\sup _{s \in T}\{s<t\}$. The graininess function $\mu(t): T \rightarrow T$ is given by $\mu(t)=\sigma(t)-t$. Here we adopt the conventions $\inf \phi=\sup T$ (i.e. $\sigma(t)=t$ if $T$ has a maximum element $t$ ), and $\sup \phi=\inf T$ (i.e. $\rho(t)=t$ if $T$ has a minimum element $t$ ). For notational purpose, the intersection of a real interval $[a, b]$ with a time scale $T$ is denoted by $[a, b] \cap T:=[a, b]_{T}$. A point $t \in T$ is right scattered if $\sigma(t)>t$ and right dense if $\sigma(t)=t$. A point $t \in T$ is left scattered if $\rho(t)<t$ and left dense if $\rho(t)=t$. If $t$ is both left scattered and right scattered, we say $t$ is isolated. If $t$ is both left dense and right dense, we say that $t$ is dense. The set $T^{k}$ is defined as follows: if $T$ has a left scattered maximum $m$, then $T^{k}=T-\{m\}$; otherwise $T^{k}=T$. If $f: T \rightarrow \mathbf{R}$ is a function, then the composition $f(\sigma(t))$ is often denoted by $f^{\sigma}(t)$.

For $f: T \rightarrow \mathbf{R}$ and $t \in T^{k}$, define $f^{\Delta}(t)$ as the number (when it exists), with the property that, for any $\epsilon>0$, there exists a neighborhood $U$ of $t$ such 
that

$$
\left|[f(\sigma(t))-f(s)] f^{\Delta}(t)[\sigma(t)-s]\right| \leq \epsilon|\sigma(t)-s|, \quad \text { for all } s \in U .
$$

The function $f^{\Delta}: T^{k} \rightarrow \mathbf{R}$ is called the delta derivative or the Hilger derivative of $f$ on $T^{k}$. We say $f$ is delta differentiable on $T^{k}$ provided $f^{\Delta}(t)$ exists for all $t \in T^{k}$.

The following theorem establishes several important observations regarding delta derivatives.

Theorem 2.1 Suppose $f: T \rightarrow \mathbf{R}$ and $t \in T^{k}$.

(i) If $f$ is delta differentiable at $t$, then $f$ is continuous at $t$.

(ii) If $f$ is continuous at $t$ and $t$ is right scattered, then $f$ is delta differentiable at $t$ and

$$
f^{\Delta}(t)=\frac{f(\sigma(t))-f(t)}{\mu(t)} .
$$

(iii) If $t$ is right dense, then $f$ is delta differentiable at $t$ if and only if $\lim _{s \rightarrow t} \frac{f(t)-f(s)}{t-s}$ exists. In this case,

$$
f^{\Delta}(t)=\lim _{s \rightarrow t} \frac{f(t)-f(s)}{t-s} .
$$

(iv) If $f$ is delta differentiable at $t$, then $f(\sigma(t))=f(t)+\mu(t) f^{\Delta}(t)$.

Note that $f^{\Delta}(t)$ is precisely $f^{\prime}(t)$ from the usual calculus when $T=\mathbf{R}$. On the other hand, $f^{\Delta}=\Delta f=f(t+1)-f(t)$ (i.e. the forward difference operator) on the time scale $T=\mathbf{R}$. These are but two very special (and rather simple) examples of time scales. Moreover, the realm of differential equations and difference equations can now viewed as but special, particular cases of more general dynamic equations on time scales, i.e. equations involving the delta derivative(s) of some unknown function.

A function $f: T \rightarrow \mathbf{R}$ is rd-continuous if $f$ is continuous at every right dense point $t \in T$, and its left hand limit exists at each left dense point. The set of rd-continuous functions $f: T \rightarrow \mathbf{R}$ will be denoted by $C_{r d}=C_{r d}(T)=$ $C_{r d}(T, \mathbf{R})$. A function $f: T \rightarrow \mathbf{R}$ is called a (delta) antiderivative of $f: T \rightarrow \mathbf{R}$ provided $F^{\Delta}(t)=f(t)$ holds for all $t \in T^{k}$. The Cauchy integral or definite integral is given by $\int_{a}^{b} f(t) \Delta t=F(b)-F(a)$, for all $a, b \in T$, where $F$ is any (delta) antiderivative $f$. Suppose that $\sup T=\infty$. Then the improper integral is defined to by $\int_{a}^{\infty} f(t) \Delta t=\left.\lim _{b \rightarrow \infty} F(t)\right|_{a} ^{b}$ for all $a \in T$.

Theorem 2.2 (Existence of antiderivatives). 
(i) Every rd-continuous function has an antiderivative. If $t_{0} \in T$, then $F(t)=\int_{t_{0}}^{t} f(\tau) \Delta \tau, \quad \tau \in T$, is an antiderivative of $f$.

(ii) If $f \in C_{r d}$ and $t \in T^{k}$, then $\int_{t}^{\sigma(t)} f(\tau) \Delta(\tau)=f(t) \mu(t)$.

(iii) Suppose $a, b \in T$ and $f \in C_{r d}$.

(a) If $T=\mathbf{R}$, then $\int_{a}^{b} f(t) \Delta(t)=\int_{a}^{b} f(t) d t$ (the usual Riemann integral).

(b) If $[a, b]_{T}$ consists of only isolated points, then

$$
\int_{a}^{b} f(t) \Delta(t)= \begin{cases}\sum_{t \in[a, b)_{T}} f(t) \mu(t) & ; a<b \\ 0 & ; a=b \\ -\sum_{t \in[a, b)_{T}} f(t) \mu(t) & ; a>b\end{cases}
$$

The last result above reveals that in the continuous case, $T=\mathbf{R}$, definite integrals are the usual Riemann integrals from calculus. When $T=\mathbf{Z}$, definite integrals correspond to definite sums from the difference calculus; see [8].

\subsection{The Hilger's complex plane}

For $h>0$, define the Hilger complex numbers, the Hilger real axis, the Hilger alternating axis, and the Hilger imaginary circle by

$$
\begin{array}{ll}
C_{h}:=\left\{z \in \mathbf{C}: z \neq-\frac{1}{h}\right\}, \quad R_{h}:=\left\{z \in \mathbf{R}: z>-\frac{1}{h}\right\}, \\
A_{h}:=\left\{z \in \mathbf{R}: z<-\frac{1}{h}\right\}, & I_{h}:=\left\{z \in \mathbf{C}:\left|z+\frac{1}{h}\right|=\frac{1}{h}\right\},
\end{array}
$$

Respectively. For $h=0$, let $C_{0}=\mathbf{C}, R_{0}=\mathbf{R}, A_{0}=\emptyset$ and $I_{0}=i \mathbf{R}$.

Let $h>0$ and $z \in C_{h}$. The Hilger real part of $z$ is defined by $\operatorname{Re}_{h}(z):=\frac{|z h+1|-1}{h}$, and the Hilger imaginary part of $z$ is defined by $\operatorname{Im}_{h}(z):=\frac{\operatorname{Arg}(z h+1)}{h}$, where $\operatorname{Arg}(z)$ denotes the principal argument of $z$ (i.e. $-\pi<\operatorname{Arg}(z) \leq \pi)$.

For $h>0$, define the strip $Z_{h}:=\left\{z \in \mathbf{C}:-\frac{\pi}{h}<\operatorname{Im}(z) \leq \frac{\pi}{h}\right\}$, and for $h=0$, set $Z_{0}=\mathbf{C}$. Then we can define the cylinder transformation $\xi_{h}: C_{h} \rightarrow$ $Z_{h}$ by

$$
\xi_{h}(z)=\frac{1}{h} \log (1+z h), \quad z>0,
$$

where $\log$ is the principal logarithm function. When $h=0$, we define $\xi_{0}(z)=$ $z$, for all $z \in \mathbf{C}$. It then follows that the inverse cylinder transformation $\xi_{h}^{-1}: Z_{h} \rightarrow C_{h}$ is given by

$$
\xi_{h}^{-1}(z)=\frac{e^{z h}-1}{h}
$$

Since the graininess may not be constant for a given time scale, we will interchangeably subscript various quantities (such as $\xi$ and $\xi^{-1}$ ) with $\mu=\mu(t)$ 
instead of $h$ to reflect this.

\subsection{Generalized exponential functions}

The function $p: T \rightarrow \mathbf{R}$ is regressive if $1+\mu(t) p(t) \neq 0$ for all $t \in T^{k}$, and this concept motivates the definition of the following sets:

$$
\begin{aligned}
& \mathcal{R}=\left\{p: T \rightarrow \mathbf{R}: p \in C_{r d}(T) \text { and } 1+\mu(t) p(t) \neq 0, \forall t \in T^{k}\right\} \\
& \mathcal{R}^{+}=\left\{p \in \mathbf{R}: 1+\mu(t) p(t)>0, \forall t \in T^{k}\right\} .
\end{aligned}
$$

The function $p: t \rightarrow \mathbf{R}$ is uniformly regressive on $T$ if there exists a positive constant $\delta$ such that $0<\delta^{-1} \leq|1+\mu(t) p(t)|, t \in T^{k}$. A matrix is regressive if and only if all of its eigenvalues are in Equivalently, the matrix $A(t)$ is regressive if and only if $I+\mu(t) A(t)$ is invertible for all $t \in T^{k}$.

If $p \in \mathcal{R}$, then we define the generalized time scale exponential function by

$$
e_{p}(t, s)=\exp \left(\int_{s}^{t} \xi_{\mu(\tau)}(p(\tau)) \Delta(\tau)\right) \quad \text { for all } s, t \in T .
$$

The following theorem is a compilation of properties of $e_{p}\left(t, t_{0}\right)$ (some of which are counterintuitive) that we need in the main body of the paper.

Theorem 2.3 The function has the following properties:

(i) If $p \in \mathcal{R}$ then $e_{p}(t, r) e_{p}(r, s)=e_{p}(t, s)$ for all $r, s, t \in T$.

(ii) $e_{p}(\sigma(t), s)=(1+\mu(t) p(t)) e_{p}(t, s)$.

(iii) If $p \in \mathcal{R}^{+}$, then $e_{p}\left(t, t_{0}\right)>0$ for all $t \in T$.

(iv) If $1+\mu(t) p(t)<0$ for some $t \in T^{k}$, then $e_{p}\left(t, t_{0}\right) e_{p}\left(\sigma(t), t_{0}\right)<0$.

(v) If $T=\mathbf{R}$ then $e_{p}(t, s)=e^{\int_{s}^{t} p(\tau) d \tau}$. Moreover, if $p$ is constant, then $e_{p}(t, s)=e^{p(t-s)}$.

(vi) If $T=\mathbf{Z}$, then $e_{p}(t, s)=\prod_{\tau=s}^{t-1}(1+p(\tau))$. Moreover, if $T=h \mathbf{Z}$, with $h>0$ and $p$ is constant, then $e_{p}(t, s)=(1+h p)^{(t-s) / h}$.

If $p \in \mathcal{R}$ is rd-continuous, then the dynamic equation

$$
y^{\Delta}(t)=p(t) y(t)
$$

is called regressive.

Theorem 2.4 Let $t_{0}=T$ and $y\left(t_{0}\right)=y_{0} \in \mathbf{R}$. Then the regressive IVP (2) has a unique solution $y: T \rightarrow \mathbf{R}^{n}$ given by

$$
y(t)=e_{p}\left(t, t_{0}\right) y_{0}
$$


If $p \in \mathcal{R}$ and $f: T \rightarrow \mathbf{R}$ is rd-continuous, then the dynamic equation

$$
y^{\Delta}(t)=p(t) y(t)+f(t)
$$

is called regressive.

Theorem 2.5 (Variation of constants). Let $t_{0}=T$ and $y\left(t_{0}\right)=y_{0} \in \mathbf{R}$. Then the regressive IVP (3) has a unique solution $y: T \rightarrow \mathbf{R}^{n}$ given by

$$
y(t)=e_{p}\left(t, t_{0}\right) y_{0}+\int_{t_{0}}^{t} e_{p}(t, \sigma(\tau)) f(\tau) \Delta \tau .
$$

We say the $n \times 1$-vector-valued system

$$
y^{\Delta}(t)=A(t) y(t)+f(t)
$$

is regressive provided $A \in \mathcal{R}$ and $f: T \rightarrow \mathbf{R}^{n}$ is a rd-continuous vector-valued function. Let $t_{0} \in T$ and assume that $A$ is a $n \times n$-matrix-valued function. The unique matrix-valued solution to the IVP

$$
Y^{\Delta}(t)=A(t) Y(t), \quad Y\left(t_{0}\right)=I_{n},
$$

where $I_{n}$ is the $n \times n$-identity matrix, is called the transition matrix and it is denoted by $\Phi_{A}\left(t, t_{0}\right)$. In this paper, we denote the solution to (5) as $\Phi_{A}\left(t, t_{0}\right)$ when $A(t)$ is time varying and denoted the solution as $e_{A}\left(t, t_{0}\right) \equiv \Phi_{A}\left(t, t_{0}\right)$ (the matrix exponential), as in [1]) only when $A(t) \equiv A$ is a constant matrix. Also, if $A(t)$ is a function on $T$ and the time scale matrix exponential function is a function on some other time scale $\mathbf{S}$, then $A(t)$ is constant with respect to $e_{A(t)}(\tau, s)$, for all $\tau, s \in \mathbf{S}$ and $t \in T$. The following theorem lists some properties of the transition matrix.

Theorem 2.6 Suppose $A, B \in \mathcal{R}$ are matrix-valued function on $T$.

(i) Then the semigroup property $\Phi_{A}(t, r) \Phi_{A}(r, s)=\Phi_{A}(t, s)$ is satisfied for all $r, s, t \in T$.

(ii) $\Phi_{A}(\sigma(t), s)=(I+\mu(t) A(t)) \Phi_{A}(t, s)$.

(iii) If $T=\mathbf{R}$, then $A$ is constant, then $\Phi_{A}(t, s)=e_{A}(t, s)=e^{A(t-s)}$.

(iv) If $T=h \mathbf{Z}$, with $h>0$ and $A$ is constant, then $\Phi_{A}(t, s)=e_{A}(t, s)=$ $(1+h A)^{(t-s) / h}$.

We now present a theorem that guarantees a unique solution to the regressive $n \times 1$-vector-valued dynamic IVP (4). 
Theorem 2.7 (Variation of constants). Let $t_{0}=T$ and $y\left(t_{0}\right)=y_{0} \in \mathbf{R}^{n}$. Then the regressive IVP (4) has a unique solution $y: T \rightarrow \mathbf{R}^{n}$ given by

$$
y(t)=\Phi_{A}\left(t, t_{0}\right) y_{0}+\int_{t_{0}}^{t} \Phi_{A}(t, \sigma(\tau)) f(\tau) \Delta \tau .
$$

\section{Main results}

In this section we consider the existence of solution for the time scale system

$$
\begin{gathered}
y^{\nabla}=A(t) y(t)+F(t, y(t)) \text { for } t \in\left[t_{0}, \infty\right)_{T} . \\
\text { with } M y\left(t_{0}\right)+N y\left(t_{1}\right)=\alpha
\end{gathered}
$$

which can be extended to delta case and problem

$$
y^{\nabla}=A(t) y(t)+F\left(t, y(\rho(t)) \text { for } t \in\left[t_{0}, \infty\right)_{T} .\right.
$$

with the same conditions. Here $A:\left[t_{0}, t_{1}\right] \rightarrow B\left(R^{n}\right)$ is ld-continuous and regressive and $M$ and $N$ square matrix of order $n$. Here we consider necessary and sufficient conditions for the existence of a solution to $y^{\Delta}=A(t) y(t)+f(t)$ with (7) is that if

$$
D=M \Phi_{A}\left(t_{0}, t_{0}\right)+N \Phi_{A}\left(t_{1}, t_{0}\right)
$$

is nonsingular. In this case, the solution can be written as

$$
\left.y(t)=\Phi_{A}\left(t, t_{0}\right) D^{-1}\left(\alpha-N \int_{t_{0}}^{t_{1}} \Phi_{A}(t, \rho(\tau)) f(\tau) \Delta \tau\right)+\int_{t_{0}}^{t_{1}} \Phi_{A}(t, \rho(\tau)) f(\tau) \Delta \tau\right) .
$$

Above result together with a fixed point result of Lery-Schauder type can be established existence results for the nonlinear system (6),(7). For this we state here the fixed point result [8] which will use in this section.

Theorem 3.1 Let $C$ be a convex subset of a Banach space $E$, and let $U$ be an open subset of $C$ with $0 \in U$. Then every compact, continuous map $\Gamma: \bar{U} \rightarrow C$ has at least one of the following two properties:

(i) $N$ has a fixed point in $\bar{U}$.

(ii) There is a $x \in \partial U$ and $\lambda \in(0,1)$ with $x=\lambda \Gamma(x)$.

Now we consider the system

$$
\begin{gathered}
y^{\nabla}=A(t) y(t)+F\left(t, y(t) \text { for } t \in\left[t_{0}, t_{1}\right]_{T} .\right. \\
\text { with } \quad M y\left(t_{0}\right)+N y\left(t_{1}\right)=\alpha
\end{gathered}
$$

Here $\mu>0$ is a constant.

Theorem 3.2 Let $A:\left[t_{0}, t_{1}\right] \rightarrow B\left(R^{n}\right)$ is ld-continuous and regressive, $f:$ $\left[t_{0}, t_{1}\right] \times R^{n} \rightarrow R^{n}$ be continuous. Assume $\sup _{t \in\left[t_{0}, t_{1}\right]}\left\|\Phi_{A}\left(t, t_{0}\right)\right\|<\infty$ and $D=$ $M \Phi_{A}\left(t_{0}, t_{0}\right)+N \Phi_{A}\left(t_{1}, t_{0}\right)$ is nonsingular. Suppose the following conditions are satisfied: 
(i) There exists a continuous, nondecreasing function $\psi:[0, \infty) \rightarrow[0, \infty)$ with $\|f(s, x(s))\| \leq r(s) \psi(\|x(s)\|)+q(s), r(s) \geq 0, q(s) \geq 0$ for all $x \in$ $B\left[t_{0}, t_{1}\right]$ and $s \in B\left[t_{0}, t_{1}\right]$

(ii) $\sup _{t \in\left[t_{0}, t_{1}\right]} \int_{t_{0}}^{t_{1}}\left\|\Phi_{A}(t, \rho(\tau))\right\| r(s) \nabla s \equiv W<\infty$

(iii) $\sup _{t \in\left[t_{0}, t_{1}\right]} \int_{t_{0}}^{t_{1}}\left\|\Phi_{A}(t, \rho(\tau))\right\| q(s) \nabla s \equiv P<\infty$

(iv) $\mu_{0} \geq 0$ satisfies here $\sup _{t \in(0, \infty)}\left(\frac{x}{E_{0} \mu_{0} \psi(x)+E_{1} \mu_{0}+E_{2}}\right)>1$ here

$E_{0}=W+W Q\left\|D^{-1}\right\|\|N\|, E_{1}=P+P Q\left\|D^{-1}\right\|\|N\|, E_{2}=Q\left\|D^{-1}\right\|\|\alpha\|$ and $Q=\left\|\Phi_{A}\right\|=\sup _{t \in\left[t_{0}, t_{1}\right]}\left\|\Phi_{A}(t)\right\|$

If $0 \leq \mu \leq \mu_{0}$ then (10),(11)(for every $\alpha \in R^{n}$ ) has a solution in $B\left[t_{0}, t_{1}\right]$.

Proof. Fix $0 \leq \mu \leq \mu_{0}$. Let $M_{0}>0$ satisfy

$$
\left.\frac{M_{0}}{E_{0} \mu_{0} \psi\left(M_{0}\right)+E_{1} \mu_{0}+E_{2}}\right)>1
$$

Consider the operator $T: D\left[t_{0}, t_{1}\right] \rightarrow D\left[t_{0}, t_{1}\right]$ defined by $T(\bar{x})(t)=\bar{y}(t)$ (here $\bar{x} \in D\left[t_{0}, t_{1}\right]$ ) where for $t \in\left[t_{0}, t_{1}\right]$,

$$
\begin{aligned}
y(t)= & \Phi_{A}\left(t, t_{0}\right) D^{-1} \alpha+\int_{t_{0}}^{t} \Phi_{A}(t, \rho(s)) \mu f(s, \bar{x}(s)) \nabla s . \\
& -\Phi_{A}\left(t, t_{0}\right) D^{-1} N\left[\int_{t_{0}}^{t} \Phi_{A}(t, \rho(s)) \mu f(s, \bar{x}(s)) \nabla s\right]
\end{aligned}
$$

We wish to apply theorem 3.1. First we show $T: D\left[t_{0}, t_{1}\right] \rightarrow D\left[t_{0}, t_{1}\right]$ is continuous. To see this let $\bar{x}, \bar{x}_{n} \in D\left[t_{0}, t_{1}\right]$ and define $\bar{y}=T(\bar{x}(t))$ and $\bar{y}_{n}=$ $T\left(\bar{x}_{n}(t)\right)$. Then

$$
\begin{gathered}
\left\|T(\bar{x})-T\left(\bar{x}_{n}\right)\right\|_{D\left[t_{0}, t_{1}\right]}=\left\|\bar{y}-\bar{y}_{n}\right\|_{D\left[t_{0}, t_{1}\right]}=\left\|\bar{y}-\bar{y}_{n}\right\|=\sup _{t \in\left[t_{0}, t_{1}\right]}\left\|\bar{y}-\bar{y}_{n}\right\| \\
\leq \operatorname{ssup}_{t \in\left[t_{0}, t_{1}\right]} \int_{t_{0}}^{t}\left\|\Phi_{A}(t, \rho(s))\right\|\left\|f(s, \bar{x}(s))-f\left(s, \bar{x}_{n}(s)\right)\right\| \nabla s \\
+\mu Q\left\|D^{-1}\right\|\|N\| \sup _{t \in\left[t_{0}, t_{1}\right]} \int_{t_{0}}^{t}\left\|\Phi_{A}(t, \rho(s))\right\|\left\|f(s, \bar{x}(s))-f\left(s, \bar{x}_{n}(s)\right)\right\| \nabla s
\end{gathered}
$$

It is also easy to see that $\mid \bar{x}-\bar{x}_{n} \|_{D\left[t_{0}, t_{1}\right]} \rightarrow 0$ implies

$$
\rho=\sup _{t \in\left[t_{0}, t_{1}\right]} \int_{t_{0}}^{t}\left\|\Phi_{A}(t, \rho(s))\right\|\left\|f(s, \bar{x}(s))-f\left(s, \bar{x}_{n}(s)\right)\right\| \nabla s \rightarrow 0
$$


as $n \rightarrow \infty$. This together with

$$
\begin{array}{r}
\left\|T(\bar{x})-T\left(\bar{x}_{n}\right)\right\|_{D\left[t_{0}, t_{1}\right]}<\mu \rho \sup _{t \in\left[t_{0}, t_{1}\right]} \int_{t_{0}}^{t_{1}}\left\|\Phi_{A}(t, \rho(s))\right\| \nabla s \\
+\mu Q\left\|D^{-1}\right\|\|N\| \rho \sup _{t \in\left[t_{0}, t_{1}\right]} \int_{t_{0}}^{t_{1}}\left\|\Phi_{A}(t, \rho(s))\right\| \nabla s
\end{array}
$$

guarantees that $T: D\left[t_{0}, t_{1}\right] \rightarrow D\left[t_{0}, t_{1}\right]$ is continuous. Next we show $T$ is completely continuous. Let $\Omega$ be a bounded subset of $D\left[t_{0}, t_{1}\right]$, so there exists $r_{0}>0$ with $\mid \bar{x} \|_{D\left[t_{0}, t_{1}\right]}<r_{0}$ for each $\bar{x} \in \Omega$. Let $\bar{y}=T(\bar{x})(t)$ for each $\bar{x} \in \Omega$.

Then

$$
\begin{aligned}
\| T\left(\bar{x} \|_{D\left[t_{0}, t_{1}\right]}\right. & =\|\bar{y}\|_{D\left[t_{0}, t_{1}\right]}=\|\bar{y}\| \\
& \leq Q\left\|D^{-1}\right\|\|\alpha\|+\mu \sup _{t \in\left[t_{0}, t_{1}\right]} \int_{t_{0}}^{t_{1}}\left\|\Phi_{A}(t, \rho(s))\right\|\|f(s, \bar{x}(s))\| \nabla s \\
& +\mu Q\left\|D^{-1}\right\|\|N\| \sup _{t \in\left[t_{0}, t_{1}\right]} \int_{t_{0}}^{t_{1}}\left\|\Phi_{A}(t, \rho(s))\right\|\|f(s, \bar{x}(s))\| \nabla s \\
& \leq Q\left\|D^{-1}\right\|\|\alpha\|+\mu\left[W \psi\left(r_{0}\right)+P\right]+\mu Q\left\|D^{-1}\right\|\|N\|\left[W \psi\left(r_{0}\right)+P\right]
\end{aligned}
$$

and for $z_{1}, z_{2} \in\left[t_{0}, t_{1}\right]$ we have

$$
\begin{aligned}
\left\|T(\bar{x})\left(z_{1}\right)-T(\bar{x})\left(z_{2}\right)\right\| & \leq\left\|\bar{y}\left(z_{1}\right)-\bar{y}\left(z_{2}\right)\right\|=\left\|y\left(z_{1}\right)-y\left(z_{2}\right)\right\| \\
& \leq\left\|\left[\Phi_{A}\left(z_{1}\right)-\Phi_{A}\left(z_{2}\right)\right] D^{-1} \alpha\right\| \\
& +\| \int_{t_{0}}^{z_{1}} \Phi_{A}\left(z_{1}, \rho(s)\right) \mu f(s, \bar{x}(s)) \nabla s \\
& -\int_{t_{0}}^{z_{2}} \Phi_{A}\left(z_{2}, \rho(s)\right) \mu f(s, \bar{x}(s)) \nabla s \| \\
& +\left\|\left[\Phi_{A}\left(z_{1}\right)-\Phi_{A}\left(z_{2}\right)\right] D^{-1} N\left[\int_{t_{0}}^{t} \Phi_{A}(t, \rho(s)) \mu f(s, \bar{x}(s)) \nabla s\right]\right\| \\
& \leq\left\|\left[\Phi_{A}\left(z_{1}\right)-\Phi_{A}\left(z_{2}\right)\right] D^{-1} \alpha\right\| \\
& +\int_{t_{0}}^{t_{1}}\left\|\Phi_{A}\left(z_{1}, \rho(s)\right)-\Phi_{A}\left(z_{2}, \rho(s)\right)\right\| \mu\left[r(s) \psi\left(r_{0}\right)+q(s)\right] \nabla s \\
& +\left|\int_{z_{1}}^{z_{2}}\left\|\Phi_{A}\left(z_{2}, \rho(s)\right)\right\| \mu\left[r(s) \psi\left(r_{0}\right)+q(s)\right] \nabla s\right| \\
& +\left\|\Phi_{A}\left(z_{1}\right)-\Phi_{A}\left(z_{2}\right)\right\|\left\|D^{-1}\right\|\|N\| \\
& \times \sup _{t \in\left[t_{0}, t_{1}\right]} \int_{t_{0}}^{t_{1}}\left\|\Phi_{A}(t, \rho(s))\right\| \mu\left[r(s) \psi\left(r_{0}\right)+q(s)\right] \nabla s
\end{aligned}
$$

The Arzela-Ascoli theorem [4] guarantees that $T: D\left[t_{0}, t_{1}\right] \rightarrow D\left[t_{0}, t_{1}\right]$ is completely continuous. Now assume the equation

$$
\bar{x}(t)=\lambda T(\bar{x}(t))
$$


has a solution in $D\left[t_{0}, t_{1}\right]$ for some $0<\lambda<1$. Then

$$
\begin{aligned}
\|\bar{x}\|_{D\left[t_{0}, t_{1}\right]}=\|\bar{x}\| & \leq Q\left\|D^{-1}\right\|\|\alpha\|+\mu \sup _{t \in\left[t_{0}, t_{1}\right]} \int_{t_{0}}^{t_{1}}\left\|\Phi_{A}(t, \rho(s))\right\|\|f(s, \bar{x}(s))\| \nabla s \\
& +\mu Q\left\|D^{-1}\right\|\|N\| \sup _{t \in\left[t_{0}, t_{1}\right]} \int_{t_{0}}^{t_{1}}\left\|\Phi_{A}(t, \rho(s))\right\|\|f(s, \bar{x}(s))\| \nabla s \\
& \leq Q\left\|D^{-1}\right\|\|\alpha\|+\mu\left[W \psi\left(\|\bar{x}\|_{D\left[t_{0}, t_{1}\right]}\right)+P\right] \\
& +\mu Q\left\|D^{-1}\right\|\|N\|\left[W \psi\left(\|\bar{x}\|_{D\left[t_{0}, t_{1}\right]}\right)+P\right] \\
& =E_{2}+E_{1} \mu+E_{0} \mu \psi\left(\left\|\bar{x}_{D\left[t_{0}, t_{1}\right]}\right\|\right)
\end{aligned}
$$

and so

$$
\left.\frac{\|\bar{x}\|_{D\left[t_{0}, t_{1}\right]}}{E_{0} \mu_{0} \psi\left(\|\bar{x}\|_{D\left[t_{0}, t_{1}\right]}\right)+E_{1} \mu_{0}+E_{2}}\right) \leq 1
$$

This together with (12) implies

$$
\|\bar{x}\|_{D\left[t_{0}, t_{1}\right]} \neq M_{0}
$$

for any solution $\bar{x} \in D\left[t_{0}, t_{1}\right]$ to (13). Apply theorem 3.1 with

$$
U=\left\{\bar{x} \in D\left[t_{0}, t_{1}\right]:\|\bar{x}\|_{D\left[t_{0}, t_{1}\right]}<M_{0}\right\}, C=E=D\left[t_{0}, t_{1}\right]
$$

to deduce (note (ii) in theorem 3.1 can not occur because of (14)) that $\mathrm{T}$ has a fixed point $\bar{x}$ i.e. $\bar{x}(t)=T(\bar{x}(t))$ with $\|\bar{x}\|_{D\left[t_{0}, t_{1}\right]}<M_{0}$. So for $t \in\left[t_{0}, t_{1}\right]$ we can have

$$
\begin{aligned}
\bar{x}(t)= & \Phi_{A}\left(t, t_{0}\right) D^{-1} \alpha+\int_{t_{0}}^{t} \Phi_{A}(t, \rho(s)) \mu f(s, \bar{x}(s)) \nabla s . \\
& -\Phi_{A}\left(t, t_{0}\right) D^{-1} N\left[\int_{t_{0}}^{t} \Phi_{A}(t, \rho(s)) \mu f(s, \bar{x}(s)) \nabla s\right]
\end{aligned}
$$

In this way the result follows.

If in (i), $\psi$ is at most linear growth, then we can obtain another result of theorem 3.2 type if we use Gronwall's inequality [1].

Theorem 3.3 Let $A:\left[t_{0}, t_{1}\right] \rightarrow B\left(R^{n}\right)$ is ld-continuous and regressive, $f:$ $\left[t_{0}, t_{1}\right] \times R^{n} \rightarrow R^{n}$ be continuous. Assume $\sup _{t \in\left[t_{0}, t_{1}\right]}\left\|\Phi_{A}\left(t, t_{0}\right)\right\|<\infty$ and $D=$ $M \Phi_{A}\left(t_{0}, t_{0}\right)+N \Phi_{A}\left(t_{1}, t_{0}\right)$ is nonsingular. Suppose the following conditions are satisfied:

(i) There exists a continuous, nondecreasing function $\psi:[0, \infty) \rightarrow[0, \infty)$ with $\|f(s, x(s))\| \leq r(s) \psi(\|x(s)\|)+q(s), r(s) \geq 0, q(s) \geq 0$ for all $x \in$ $B\left[t_{0}, t_{1}\right]$ and $s \in B\left[t_{0}, t_{1}\right]$ 
(ii) $\left\{\exists b:\left[t_{0}, t_{1}\right] \rightarrow[0, \infty)\right.$ and $K>0$ with for $s \in\left[t_{0}, t_{1}\right]$, $\left\|\Phi_{A}(t, \rho(s))\right\|<K b(s)$ for all $\left.t \in\left[t_{0}, t_{1}\right]\right\}$

(iii) $\int_{t_{0}}^{t_{1}} b(s) r(s) \nabla s \equiv W_{0}<\infty$

(iv) $\int_{t_{0}}^{t_{1}} b(s) q(s) \nabla s \equiv P_{0}<\infty$

(v) $\mu_{0} \geq 0$ satisfies $\mu E_{3}\left(1+\mu K \sup _{t \in\left[t_{0}, t_{1}\right]} \int_{t_{0}}^{t_{1}} b(s) r(s) \hat{e}_{C}(t, \rho(s)) \nabla s\right)<1$;

here $C=\mu K b(s) r(s)$, and $E_{3}=Q\left\|D^{-1}\right\|\|N\| K W_{0}$ then (10),(11)(for every $\left.\alpha \in R^{n}\right)$ has a solution in $B\left[t_{0}, t_{1}\right]$.

Proof. Let $T: D\left[t_{0}, t_{1}\right] \rightarrow D\left[t_{0}, t_{1}\right]$ be as in Theorem 3.2. Essentially the same reasoning as in Theorem 3.2. guarantees that $T: D\left[t_{0}, t_{1}\right] \rightarrow D\left[t_{0}, t_{1}\right]$ is continuous and completely continuous. Now assume that the equation

$$
\bar{x}(t)=\lambda T(\bar{x}(t))
$$

has a solution in $D\left[t_{0}, t_{1}\right]$ for some $0<\lambda<1$. Then for $t \in\left[t_{0}, t_{1}\right]$ we have

$$
\begin{aligned}
\|x(t)\| & \leq Q\left\|D^{-1}\right\|\|\alpha\|+\mu K \int_{t_{0}}^{t_{1}} b(s)[r(s)\|x(s)\|+q(s)] \nabla s \\
& +\mu Q\left\|D^{-1}\right\|\|N\|+\left[W_{0}\|\bar{x}(t)\|_{D\left[t_{0}, t_{1}\right]}+P_{0}\right],
\end{aligned}
$$

and so

$$
\begin{aligned}
\|x(t)\| & \leq\left[Q\left\|D^{-1}\right\|\|\alpha\|+\mu K P_{0}+\mu E_{3}\|\bar{x}(t)\|_{D\left[t_{0}, t_{1}\right]}+\mu Q\left\|D^{-1}\right\|\|N\| K P_{0}\right] \\
& +\mu K \int_{t_{0}}^{t_{1}} b(s) r(s)\|x(s)\| \nabla s .
\end{aligned}
$$

From Gronwall's inequality [1] it follows that

$$
\|x(t)\| \leq\left(E_{4}+\mu E_{5}+E_{3}\|\bar{x}(t)\|_{D\left[t_{0}, t_{1}\right]}\right)\left(1+\mu K \int_{t_{0}}^{t} b(s) r(s) \hat{e}_{C}(t, \rho(s)) \nabla s\right)
$$

where

$$
E_{4}=Q\left\|D^{-1}\right\|\|\alpha\| \quad \text { and } \quad E_{5}=K P_{0}+Q\left\|D^{-1}\right\|\|N\| K P_{0}
$$

As a result

$$
\begin{gathered}
\|x(t)\|_{D\left[t_{0}, t_{1}\right]} \times\left\{1-\mu E_{3}\left(1+\mu K \sup _{t \in\left[t_{0}, t_{1}\right]} \int_{t_{0}}^{t_{1}} b(s) r(s) \hat{e}_{C}(t, \rho(s)) \nabla s\right)\right\} \\
\left.\leq\left(E_{4}+\mu E_{5}\right)\left(1+\mu K \sup _{t \in\left[t_{0}, t_{1}\right]} \int_{t_{0}}^{t_{1}} b(s) r(s) \hat{e}_{C}(t, \rho(s)) \nabla s\right)\right\}
\end{gathered}
$$


Thus

$$
\|x(t)\|_{D\left[t_{0}, t_{1}\right]} \leq \frac{\left.\left(E_{4}+\mu E_{5}\right)\left(1+\mu K \sup _{t \in\left[t_{0}, t_{1}\right]} \int_{t_{0}}^{t_{1}} b(s) r(s) \hat{e}_{C}(t, \rho(s)) \nabla s\right)\right\}}{\left\{1-\mu E_{3}\left(1+\mu K \sup _{t \in\left[t_{0}, t_{1}\right]} \int_{t_{0}}^{t_{1}} b(s) r(s) \hat{e}_{C}(t, \rho(s)) \nabla s\right)\right\}}
$$

Let $M_{0}=M+1$ and apply Theorem 3.1 with

$$
U=\left\{\bar{x} \in D\left[t_{0}, t_{1}\right]:\|\bar{x}\|_{D\left[t_{0}, t_{1}\right]}<M_{0}\right\}, C=E=D\left[t_{0}, t_{1}\right]
$$

to deduce that $T$ has a fixed point $\bar{x}(t)$ i.e. $\bar{x}(t)=\lambda T(\bar{x}(t))$ with

$\|x(t)\|_{D\left[t_{0}, t_{1}\right]} \leq M_{0}$. Essentially the same reasoning as in Theorem 3.2. completes the proof of Theorem 3.3.

\section{References}

[1] M. Bohner, A.Peterson,Dynamic equations on Time Scales, Birkhauser, Boston,2001.

[2] A.E.Bryson, Ho-Yu Chi, Applied optimal control optimization, estimation and control, Waltman, Massachousetts: Blaisdell,1969.

[3] B.D.O.Anderson, P.V.Kokotovich, Optimal control Problems over large time interval, Automatics, Vol.23, No.3, (1987),355-363.

[4] C.L. Chen and Y.C.Liu, Solution of two-point Boundary values Problems using differential Transformation Method, J. of optimization Theory and applications, Vol.99, No.1, 23-35.

[5] S. Deo, K.N. Murty, S. Sivasundaram, Two point Boundary values Problems associated with a systemof Generalized Matrix Differential Equations, Dynamic systems and Applications, Vol.10, (2001), 45-52.

[6] J.Goldberg, M.C.Potter, Differential equations, Prentice-Hall, Inc., (1998), 476 .

[7] Bohner M., Peterson A.,Advances in Dynamic equations on Time Scales, Birkhauser, Boston, 2003.

[8] W.Kelly, A.Peterson,Difference Equations: An introduction with Applications, Harcourt/ Academic press, Burlington,2001.

[9] Herbert B. Keller, Numerical Methods for Two-Point Boundary Value Problems, Dover Pub.Inc. New York,1992.

[10] R.P. Agarwal, M.Bohner, D. O'Regan, Time scale systems on infinite intervals, Nonlinear Analysis,(2001),837-848.

Received: September 8, 2006 